(C) 2016 IEEE. Personal use of this material is permitted. Permission from IEEE must be obtained for all other uses, in any current or future media, including reprinting/republishing this material for advertising or promotional purposes, creating new collective works, for resale or redistribution to servers or lists, or reuse of any copyrighted component of this work in other works. 


\title{
Alternative Conducted Immunity Tests
}

\author{
Soydan Çakırr ${ }^{1}$, Osman Şen ${ }^{1}$, Savaş Acak ${ }^{1}$, Marco Azpurua², Ferran Silva², Mustafa Çetintaş ${ }^{1}$ \\ ${ }^{1}$ Electromagnetic Laboratories, TUBITAK UME, Gebze, Kocaeli, Turkey \\ ${ }^{2}$ Grup de Compatibilitat Electromagnètica (GCEM) DEE ETSETB Universitat Politècnica de Catalunya, Barcelona
}

\begin{abstract}
Conducted immunity tests are always performed by the use of CDNs in laboratories in accordance with the standard EN61000-4-6. However, it is not always possible to use CDNs because of some limitations. If the EUT (Equipment Under Test) has large dimensions or high currents, it is not, most of the time, possible to send it to an EMC laboratory or to use CDNs during the test. As a consequence, usage of $\mathrm{BCI}$ probes is inevitable in industry. In this paper, we compare the laboratory setup installed with CDNs and alternative setups installed directly on mains without any CDNs in terms of loop impedances and injected loop currents. We also established a link based on the loop impedances and the injected currents on the test loops between the two setups. Finally, a first serious step was taken to establish the fundamentals of alternative conducted immunity tests based on the impedance measurements of test loops for industry.
\end{abstract}

Index Terms - Alternative, Current Probe, CDN, Conducted Immunity, EMC, High Current, Industry, Mains Impedance

\section{INTRODUCTION}

$\mathrm{T}$ he conducted immunity test is one of the major tests for industry and widely performed in laboratories in the frequency range $150 \mathrm{kHz}-80 \mathrm{MHz}$ by the use of CDNs in accordance with EN61000-4-6 [1]. The principle of the test is to induce electric and magnetic disturbance inside the EUT by applying a conducted disturbance signal in Common Mode (CM) on the EUT input and output cables. The disturbance is applied via a defined source impedance of $150 \Omega$. However, despite the fact it is not always possible to use CDNs or EM Clamps because of some limitations, there is no sufficient information in literature. A good work on deep details of conducted immunity and advantages/disadvantages of the conducted immunity test methods is perfectly presented in [2]. In addition, pitfalls and practice of conducted immunity testing were very well studied in [3]. There are also a few more researches on application of IEC61000-4-6 standard in the literature. Some of them give useful information for enhancement of standard applications such as automation of and speeding up of the tests [4-5]. The others focus on investigation and comparison of some conducted immunity test components such as EM and Bulk Current Injection (BCI) clamps in terms of tests and calibrations [6-7]. Finally, practical approach to EN61000-4-6 and variations in injected currents are studied in [8-9]. In this paper, we bring the current state-of-art research one more step further and establish the link, which is based on loop impedances and injected currents, between the laboratory conducted immunity test setups and alternative setups. While the reference setups in laboratories are always installed with CDNs, alternatives setups in industrial environments are widely installed with unknown impedances without CDNs. In addition, in our research, we were also able to take the induced impedances by BCI probe and receiving probe into account, which has not been studied in literature so far. Finally, a first serious step was taken to establish the fundamentals of alternative conducted immunity tests based on the impedance measurements of test loops. The link between the different setups was realized by means of equalization of injected currents on the test loops by using correction factors and a piece of test script written in TESEQ C3 Immunity software. For loop impedance measurements, although there are some methods in the literature, we chose the two current probe method stated in [10] for our research. In this impedance measurement method, the impedance is measured by using two current probes, a network analyzer and reference known impedances. This impedance measurement method yields the value of the unknown impedance as well as the impedance of the total loop.

We began the research firstly establishing the CM loop models of the test setups by using an EUT simulation which is a metallic box that has several settings of input impedance like in [2] and the first measurements were performed on the EUT dummies. After measuring the loop impedances of the setups, correction factors were produced by using loop impedances, comparison was performed between the theoretical correction factors and experimental correction factors in order to validate the loop impedance measurement and obtained corrections factors. In our research, while theoretical factors were calculated by using measured impedance values of reference and alternative setup loops, experimental factors were obtained by actually measuring current using a current probe in the reference and alternative setups. Further measurements were performed on an actual EUT, an SMPS. Similarly, same comparison was made between theoretical and experimental corrections factors. Then, by using the designed software script and applying the correction factors, it was shown the injected currents were equalized in both of the test loops. Finally, in order to bring the research to an advanced level, we utilized another actual EUT which is an electronic thermometer and very susceptible to injected conducted immunity disturbance signals. As with the SMPS, we began firstly with loop impedance measurements and production of correction factors based on the loop impedances. Thereafter we tested the thermometer in the test setup in controlled laboratory setup installed with 150 ohm CDNs and also in uncontrolled environment without CDNs directly on the mains, without using any correction factors to the applied power. During the tests, injected currents in both of the setup 
loops and susceptibility of the EUT were recorded for each spot frequency. As the level of susceptibility, we used the deviation caused by the injected disturbance in the shown temperature on the display of the EUT. As the last step, we applied the calculated correction factors to the industrial setup by using the designed test script and demonstrated that the equalization of injected currents and also equalization of the susceptibility level of the EUT were successfully realized in both of the setups.

\section{THEORY AND EXPERIMENTAL SETUP}

The alternative conducted immunity measurement methods in our research are completely based on the impedance measurements of loop impedances. The loop impedance measurement method that we used is stated in [10] in detail. This impedance measurement method uses a network analyzer, two current probes and one precision known impedance. It yields the value of the unknown target impedance as well as the impedance of used cables that include the effects of the used current probes and, if any, other measurement components. That means that we also took the induced impedances by the used injection clamp and receiving current clamp into account.

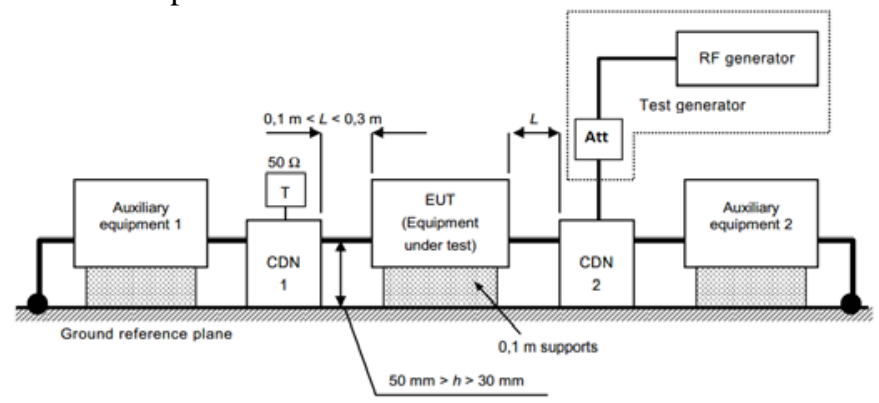

(a)

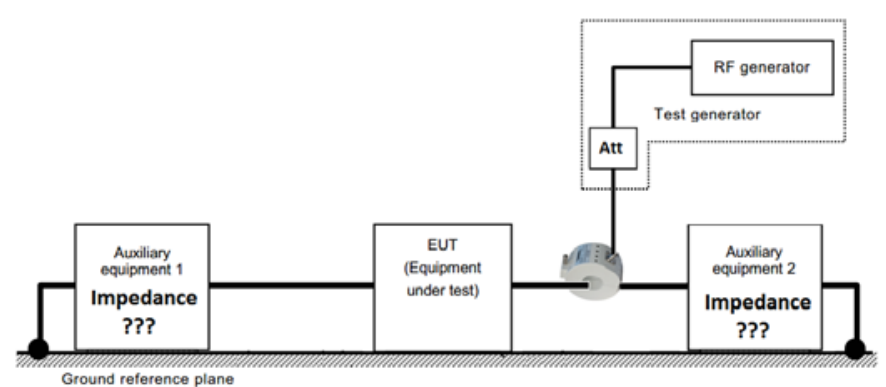

(b)

Fig 1 Conducted Immunity Setups (a) laboratory setup [1], (b) industrial setup

The standard EN61000-4-6 requires the setup presented in Fig. 1(a). On the other hand, the test setup commonly installed in industry is as depicted in Fig. 1 (b). The conducted immunity test setup circuit models installed in laboratory and in industry are also shown in Fig. 2 [2].

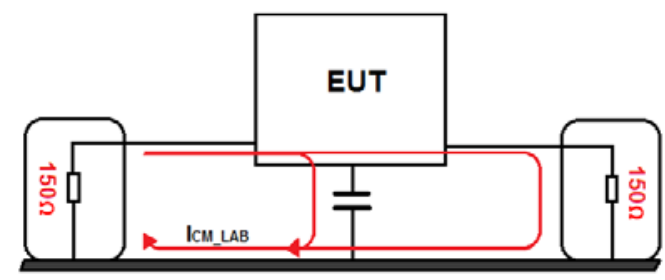

(a)

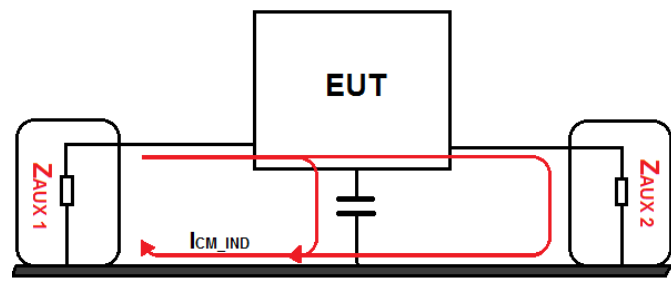

(b)

Fig 2. Conducted immunity test setups (a) laboratory setup with CDNs, (b) industrial without CDNs

As seen in Fig. 2 (a), the loop impedance includes two 150 ohm impedances and EUT on the loop. On the other hand, the setup in industry includes two unknown impedances which are shown as $\mathrm{Z}_{\mathrm{AUX} 1}$ and $\mathrm{Z}_{\mathrm{AUX} 2}$ and EUT [2]. By using the loop impedances, the theoretical correction factors can be obtained as follows;

$$
K=\frac{Z_{\text {LOOP_LABORATORY }}}{Z_{\text {LOOP_INDUSTRY }}}
$$

As measured impedances by the Network Analyzer are complex numbers, the correction factor $\mathrm{K}$ becomes a complex number. On the all graphs in this paper, we only give the module values of complex correction factors. Whenever needed, these correction factors calculated in (1) can be applied to the injected power. The injected power is obtained in the calibration phase in a test jig before the test, as stated in EN 61000-4-6.

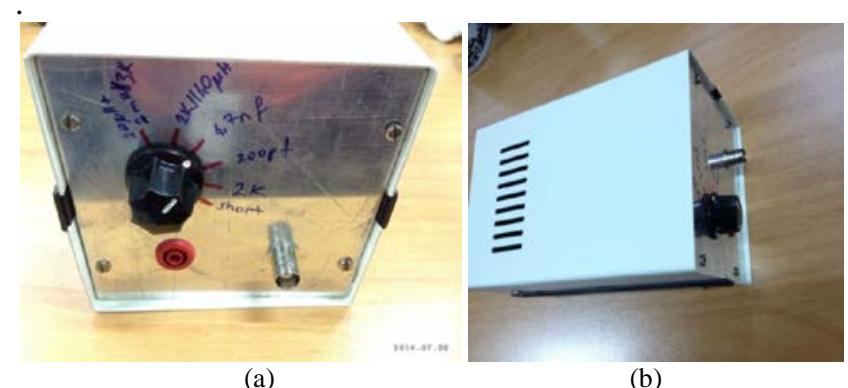

Fig 3 Dummy EUT (a) front view, (b) top view 


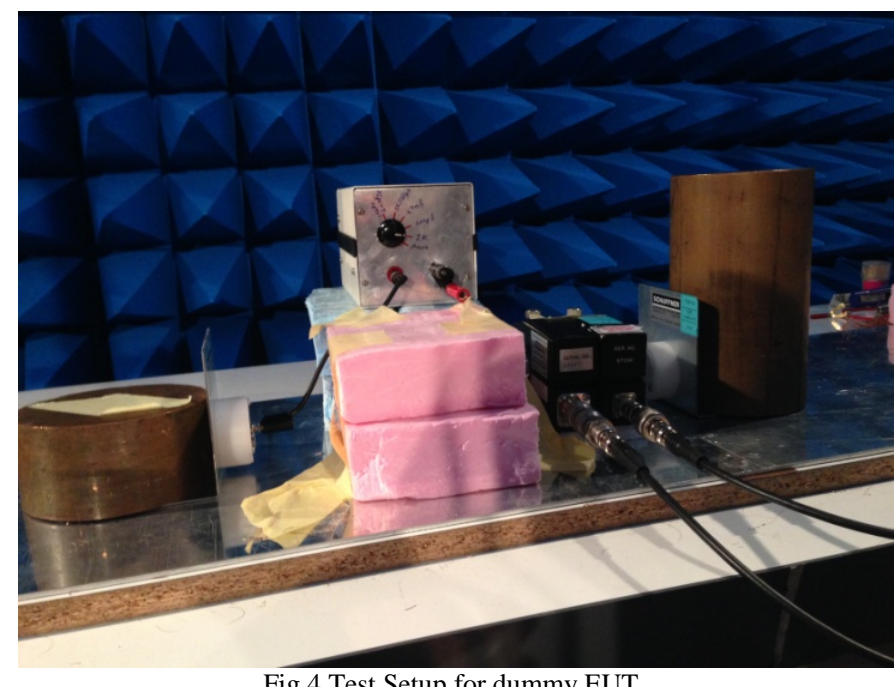

Fig 4 Test Setup for dummy EUT

After modeling the test setups, dummy EUT shown in Fig. 3 was designed with several impedance selections in order to simulate different EUT impedances. The dummy EUT includes the following impedance selections; $(20 \mu \mathrm{H}+20 \mathrm{mH})$ // $3 \mathrm{k} \Omega, 2 \mathrm{k} \Omega / / 40 \mu \mathrm{H}, 4,7 \mathrm{nF}, 200 \mathrm{pF}, 2 \mathrm{k} \Omega$ and short [2]. The dummy EUT was firstly installed on the reference setup with $150 \mathrm{ohm}$ impedances at the both sides as seen in Fig. 4 and as stipulated by the standard, then the loop impedance of the reference setup was measured by using the two-probe method described before. The two probes utilized for loop impedance measurement were also utilized for injection and receiving purposes in the test phase in order to keep consistency between loop impedance measurement and test. Thereafter a constant power level was applied to the loop and the current injected into loop was experimentally measured by the receiving probe. As the next step, with the same constant power, the dummy EUT was measured in the setup of which both sides are short-circuited to the ground, which simulates the industrial environment, together with loop impedance measurement. Finally, correction factors based on the loop impedance are calculated and they were compared to experimental results in order to check the quality of the loop impedance measurement and calculated factors.

After the measurements of the dummy EUT, we continued the measurements with an actual EUT that is an SMPS.

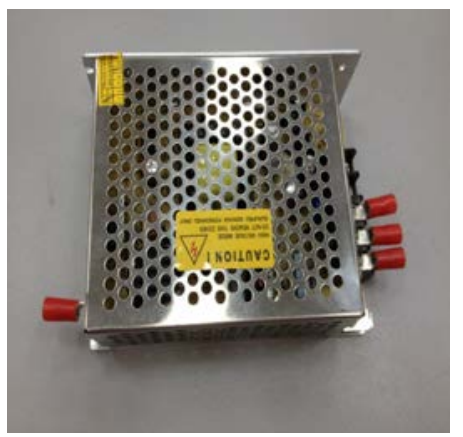

(a)

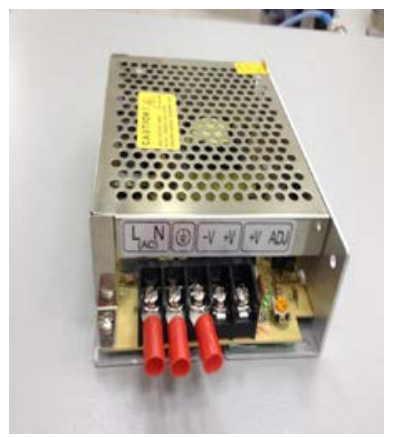

(b)
Fig 5. SMPS (a) top view, (b) front view

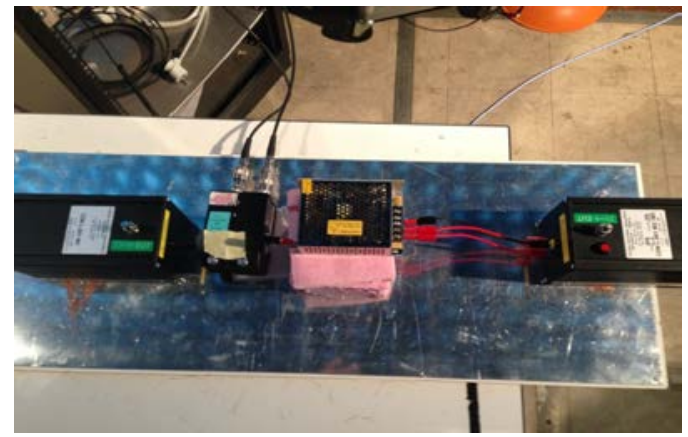

(a)

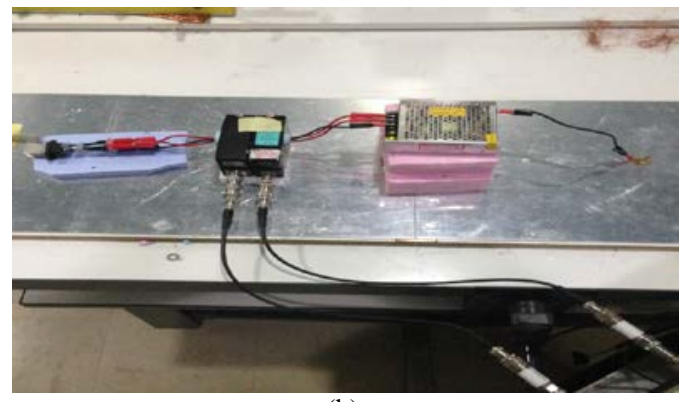

(b)

Fig 6. Conducted immunity test setups for SMPS (a) laboratory setup with CDNs, (b) industrial setup without CDNs

After the measurement of loop impedance and calculation of correction factors based on the impedance, SMPS was tested in both of the setups in turn and experimental correction factors were compared to the calculated theoretical corrections. After the theoretical correction factors are verified by comparison, these correction factors were applied to the test performed in the industrial environment simulation and equalization of injected current in both of the setups were verified. Unlike the Dummy EUT, the SMPS was electrified as intended and was directly connected to mains in one port, connected to the ground at the other port in the industrial environment simulation setup.

As a final stage of the research, we measured an electronic thermometer seen in Fig. 7, which is very susceptible to injected disturbances. The reason why we chose a susceptible device is to prove that correction factors were actually working and that the equalization of the susceptibility were actually realized along with equalization of the injected currents after the application of corrections factors. All the measurement procedure stated above for SMPS was also applied to the electronic thermometer. In addition to all, the susceptibility level of EUT, which corresponds to temperature deviation caused by injected disturbance on its display, was recorded at each test frequency during the tests. The deviation in laboratory temperature, subsequently in read value on the display of the thermometer, between the beginning and the end of the measurements in the absence of disturbance signal was recorded approximately as $0.5^{\circ}$, consequently the effect of the change in laboratory temperature on the measurement results were assumed negligible. Fig. 8 shows the installed laboratory and industrial setups with the thermometer. As seen in Fig. 8(b), the industrial setup includes the connection directly to the mains on the mains side and short-circuit connection on the auxiliary port side instead of CDNs. 


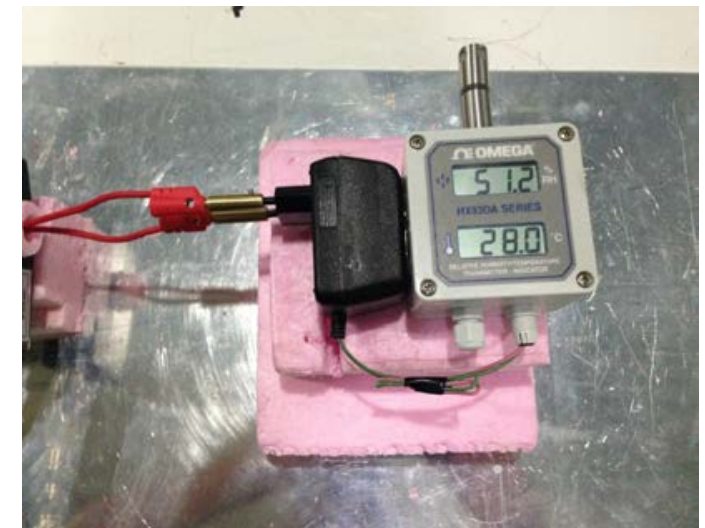

Fig 7. Electronic thermometer used as a susceptible EUT

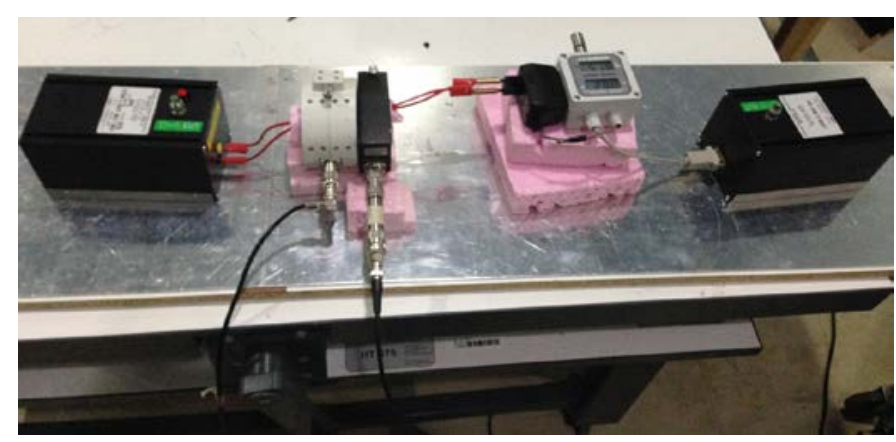

(a)

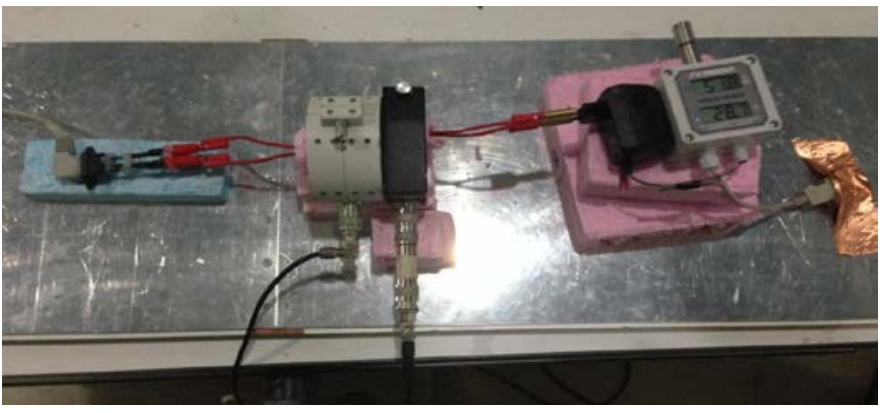

(b)

Fig 8. Conducted immunity test setups of the electronic thermometer (a) laboratory setup with CDNs, (b) industrial setup without CDNs

\section{EXPERIMENTAL RESULTS AND DISCUSSIONS}

The loop impedance measurement results and also theoretical correction factors along with experimental correction factors are presented in Fig. 9 and in Fig. 10 respectively for two switch positions chosen as examples on the impedance box given in Fig. 4. We call these switch positions SW1 and SW2 respectively. The reason why we selected these two switch positions is that we found out, after some preliminary impedance measurements, these two positions gave different loop impedance curves from the reference curve as seen in Fig.9-Fig.10. In addition, these switch positions also gave us a good opportunity of comparison between theoretical and experimental factors. Fig.9 and Fig.10 show that there is a good agreement between theoretical and experimental correction factors although there is a significant difference in loop impedances, which verifies the precision of loop impedance measurements.

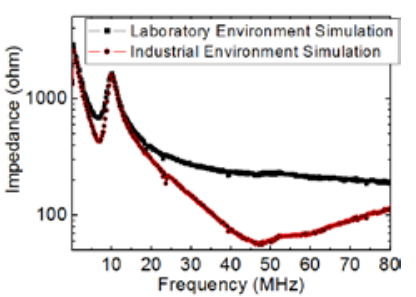

(a)

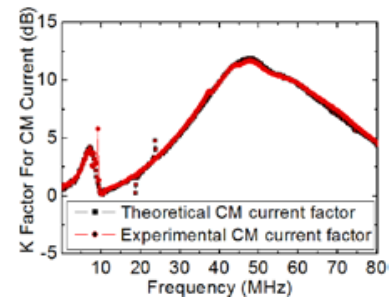

(b)
Fig 9. Results of Dummy EUT, SW1 position (a) laboratory setup with CDNs, (b) industry setup without CDNs

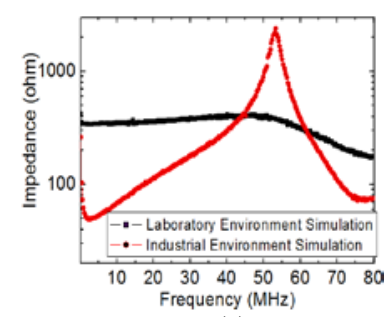

(a)

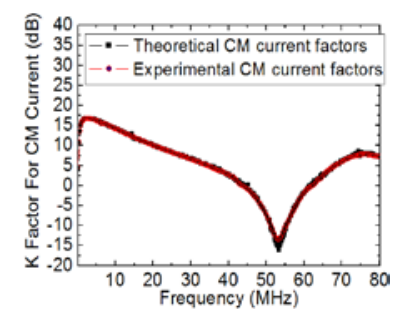

(b)
Fig 10. Results of Dummy EUT, SW2 position (a) laboratory setup with CDNs, (b) industry setup without CDNs

After verification of loop impedance measurements and calculated factors, with the dummy EUT, we continued the measurement with the SMPS. The measured loop impedances of laboratory and industrial setups and the correction factors based on these loop impedance measurement are given in Fig. 11. As seen in Fig. 11, although the loop impedance of the laboratory setup shows a very constant behavior, the loop impedance of the industrial setup shows behavior with severe resonances because the industrial setup includes the impedance of the mains. On the other hand, despite the severe resonances, there is a very good agreement between the theoretical and experimental correction factors. Besides, the injected currents on the both of the test loops are shown in Fig. 12(a) before the application of the correction factors to the injected power. The industrial injected current in Fig. 12(a) shows a similar behavior to the impedance of industrial loop in terms of resonances. After the application of correction factors to the injected power, the injected currents were almost equalized in the both of the setups except some resonances that may be caused by uncontrolled instant changes in the mains impedance.

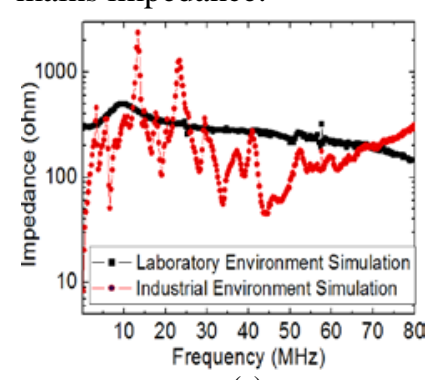

(a)

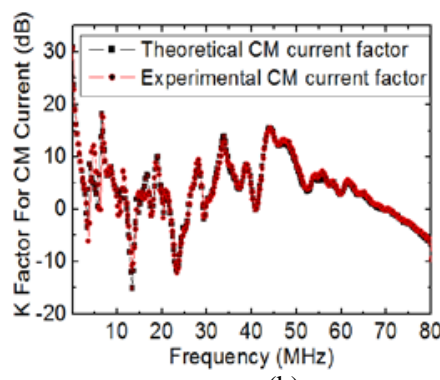

(b)
Fig 11. Results of SMPS (a) loop impedances, (b) correction factors

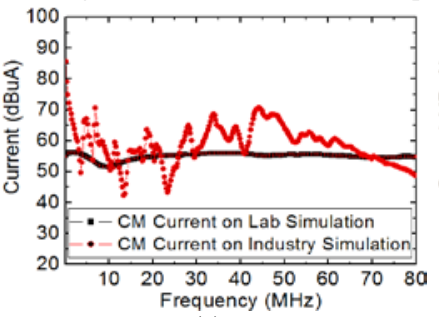

(a)

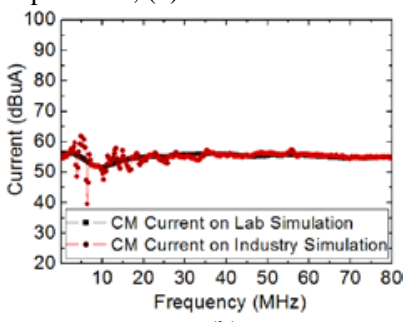

(b)
Fig 12. Injected currents for SMPS (a) without correction factors, (b) with correction factors 
In order to show the positive effects of current equalisation, we performed a final measurement on a susceptible EUT that is an electronic thermometre. The measured loop impedances of the standard and industrial setups and the correction factors based on these loop impedance measurements are given in Fig. 13. In addition, similar to SMPS, the injected currents on the both of the test loops are shown in Fig. 14(a) before the application of the correction factors to the injected power. The injected current in Fig. 14(a) shows a similar behavior to the impedance of the industrial loop in terms of resonances. After the application of the correction factors to injected power, the injected currents were almost equalized in the both of the setups as seen in Fig. 14(b). The final and more interesting results are presented in Fig. 15. Fig. 15(a) shows the susceptibility results of the thermometer before the application of correction factors to the industrial environment and the results after the application of correction factors are shown in Fig. 15(b). As seen in Fig 15 (a), the EUT shows a lot different susceptibility level in industrial setup from the laboratory setup despite the same injected power. After the application of correction factors to the input power, the susceptibility level in industrial environment became the same as the level in laboratory environment. This is the first serious step to form the link between the laboratory and industrial environment in conducted immunity tests.

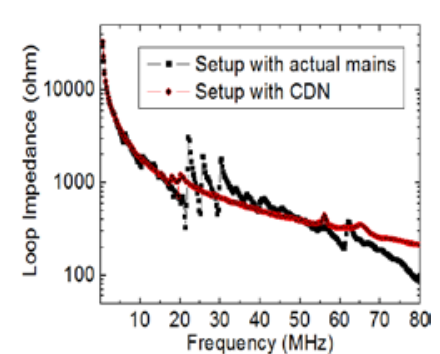

(a)

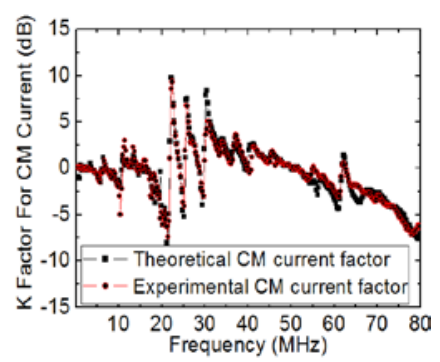

(b)
Fig 13. Results of electronic thermometer (a) loop impedance, (b) correction factors

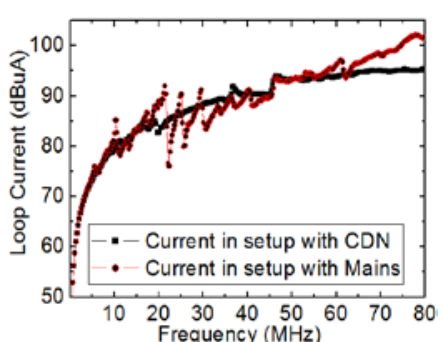

(a)

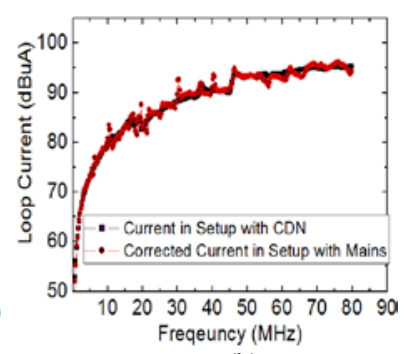

(b)
Fig 14. Injected currents for electronic thermometer (a) without correction factors, (b) with correction factors

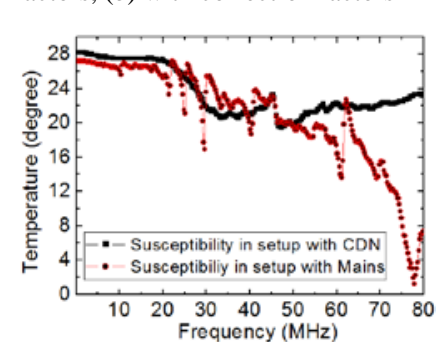

(a)

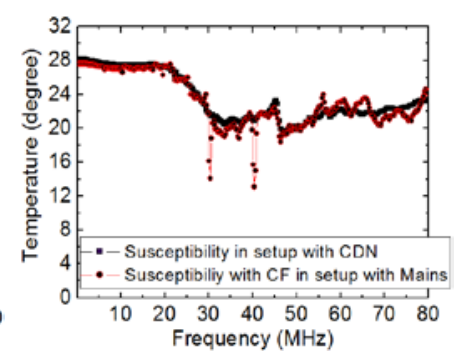

(b)
Fig 15. Susceptibility level of EUT, (a) without correction factors, (b) with correction factors
In our research, we completely focused on a link between the standard setup and the typical industrial setups in terms of the loop impedance of setups and injected currents. The research may be expanded to voltage investigations in addition to the current investigation in further researches. That will require the determination of input impedance of the EUT side to which injection is applied, which is also possible in the impedance measurement method that we utilized in this paper. This will also allow us to evaluate the effects of the voltage induced at the EUT side in addition the effects of the current.

\section{CONCLUSION}

In this research, we investigated the setups installed in laboratories and in industries in terms of loop impedances and induced currents and reached correction factors based on the loop impedances. We finally showed a link based on the correction factors on an actual EUT between the laboratory setup and industrial setup by investigating the susceptibility of an electronic thermometer in both of the setups.

\section{ACKNOWLEDGMENT}

This research is in the scope of the project IND60 "Improved EMC test methods in industrial environment” and financially supported by European Metrology Research Programme. The authors thank Bahadir Tektas for his technical support.

\section{REFERENCES}

[1] EN 61000-4-6, Electromagnetic compatibility (EMC) - Part 4-6: Testing and measurement techniques - Immunity to conducted disturbances, induced by radio-frequency fields

[2] Tim Williams, Stan Baker, "Main Report - Uncertainty of immunity measurements”, DTI-NMSPU project R2, Online: http://www.elmac.co.uk/r22b1/R22b1_mainrept.pdf

[3] Tim Williams, Stan Baker, "Pitfalls and practice of UEC 61000-4-6", DTI-NMSPU project R2, Online: http://www.elmac.co.uk/pdfs/PNP_61000-4-6.pdf

[4] K. Mourougayane, S. Karunakaran, P. Satheesan, P.K Sivakumar, "Automation of EMC tests - Software development and system integration approach”, INCEMIC 9th International Conference on Electromagnetic Interference \& Compatibility, Bangalore, India, pp. 349 - 352, 23-24 Feb. 2006.

[5] D.Pommerenke, "Methods for speeding up radiated and conducted immunity tests", IEEE International Symposium on Electromagnetic Compatibility, Washington, DC, USA, vol. 2, pp. 587 - 592, 21-25 Aug. 2000.

[6] R. Heinrich, "Investigation and comparison of different methods for EM clamp calibration”, APEMC Asia-Pacific Symposium on Electromagnetic Compatibility, Beijing, China, pp. 990 - 993, 12-16 April 2010.

[7] G. Mahesh, B. Subbarao, "Comparison of Bulk Current Injection test methods of automotive, military and civilian EMC standards", INCEMIC 10th International Conference on Electromagnetic Interference \& Compatibility, Bangalore, India, pp. 547 - 551, 26-27 Nov. 2008.

[8] J.Sroka, "Practical approach to IEC 61000-4-6 testing”, IEEE International Symposium on Electromagnetic Compatibility, Minneapolis, MN, USA, vol. 1, pp. 367 - 370, 19-23 Aug. 2002.

[9] R.Sivaramakrishnan, S.Santhakumari, R. Dhivya, S.Parthiban, L.N Charyulu, "Conducted RF immunity testing - Observed variation in the injected current", INCEMIC 10th International Conference on Electromagnetic Interference \& Compatibility, Bangalore, India, pp. 243 - 245, 26-27 Nov. 2008.

[10] Tarateeraseth V., Bo Hu; Kye Yak See, Canavero F.G., “Accurate Extraction of Noise Source Impedance of an SMPS Under Operating Conditions", IEEE Transactions on Power Electronics, Vol. 21, No.1,pp. 111-117, Jan 2010. 